\title{
Reflectance-Based Detection of Oxidizers in Ambient Air
}

Brandy J. Johnson ${ }^{\mathrm{a},}{ }^{*}$, Ray Liu ${ }^{\mathrm{b}}$, Robert C. Neblett $\mathrm{II}^{\mathrm{c}}$, Anthony P. Malanoski ${ }^{\mathrm{a}}$, Miao Xu ${ }^{\mathrm{d}}$, Jeffrey S. Erickson ${ }^{\mathrm{a}}$, Ling Zang ${ }^{\mathrm{d}}$, David A. Stenger ${ }^{\mathrm{a}}$, Martin H. Moore ${ }^{\mathrm{a}}$

${ }^{\mathrm{a} C e n t e r}$ for Bio/Molecular Science and Engineering, Naval Research Laboratory, Washington, DC 20375, USA

${ }^{\mathrm{b}}$ Thomas Jefferson High School for Science \& Technology, Alexandria, VA 22312, USA; at NRL $^{a}$ Summer 2015 through SEAP internship

${ }^{\mathrm{c}}$ Biology Department, Howard University, Washington, DC 20059, USA; at NRL ${ }^{\text {a }}$ Summer 2015 through ONR internship

${ }^{\mathrm{d}}$ Department of Materials Science and Engineering, University of Utah, Salt Lake City, UT 84108, USA

*corresponding author. Tel. 2024046100; email: brandy.white@nrl.navy.mil

\begin{abstract}
.
This study used two types of paper supported materials with a prototype, reflectance-based detector for indication of hydrogen peroxide vapor under ambient laboratory conditions. Titanyl based indicators provide detection through reaction of the indicator resulting in a dosimeter type sensor, while porphyrin based indicators provide a reversible interaction more suitable to continuous monitoring applications. These indicators provide the basis for discussion of characteristics important to design of a sensor system including the application environment and duration, desired reporting frequency, and target specificity.
\end{abstract}

KEYWORDS. peroxide, reflectance, portable sensor, vapor detection, paper-based

\section{INTRODUCTION.}

Peroxide-based homemade explosives have been identified as a threat by the US Department of Homeland Security and the US Department of Defense. Numerous online articles warn first responders of the threat these materials present, the ease of their synthesis, and their inherently unstable nature. Preparation can be as simple as mixing household chemicals, such as acetone, acid, and peroxide, making the materials favored for incorporation into improvised explosive devices (IEDs). Triacetone triperoxide (TATP), hexamethylene triperoxide diamine (HMTD), tetramethylene diperoxide dicarbamide (TMDD) and related cyclic organic peroxides are examples of specific compounds, but peroxide-based explosives can be used in either liquid or solid forms. Liquids, for example, have been used in terrorist incidents, including the 2005 attacks on transit systems in London and the foiled airline attacks of 2009.

The threat posed by these compounds has resulted in development of a wide range of detection approaches for single compounds (i.e., TATP) utilizing techniques from mass 
spectrometry through portable electrochemical approaches.[1-3] A more general method could target the hydrogen peroxide present in the liquid explosive materials and often found as an impurity and/or degradation product remaining in the solids utilized. While colorimetric detection methods for hydrogen peroxide in solution are widely available, [4-12] those available for gas phase detection are more limited.[3] A paper-based material has been reported for detection of hydrogen peroxide vapor.[13] The material relies on the interaction of peroxide with ammonium titanyl oxalate resulting in a change from white to yellow (Scheme 1). Titanium based indicators have been used by a number of groups for detection of hydrogen peroxide.[712] The recent report, however, utilizes a paper support, providing a large surface area for interaction of target with the indicator as well as an open pore network for ease of diffusion throughout the material.[13] Selectivity for hydrogen peroxide was demonstrated with no response to ethanol, methanol, acetone, tetrahydrofuran, hexane, toluene, ethyl acetate, or chloroform.

In the current study, the paper supported titanyl indicator is used with a prototype chemical sensor. The sensor hardware has previously been described for use with paper supported porphyrin indicators for the detection of alcohol vapor.[14] It relies on an array of commercially available color sensors and provides data output consisting of white, red, green, and blue color values. Data is collected in five second intervals, allowing for rapid determination of target presence. While the previous work characterized the titanium indicators under highly controlled conditions, the prototype sensor of this study provided the opportunity to look at indicator performance in the ambient environment with conditions changing in real-time.

\section{Experimental.}

For synthesis of peroxide specific indicators, ammonium titanyl oxalate monohydrate was purchased from Sigma Aldrich and used as received. The paper support materials were purchased from Whatman (Cat No. 1001 150). Loading of the paper support was accomplished using $100 \mu \mathrm{L}$ of $20 \mu \mathrm{M}$ ammonium titanyl oxalate monohydrate in water which was drop-cast onto a $2.5 \times 2.5 \mathrm{~cm}$ swatch.[13] This was followed by drying under vacuum at room temperature for $1 \mathrm{~h}$. Cobalt (CoDIX), copper (CuDIX), and nickel (NiDIX) variants of Deuteroporphyrin IX bis ethyleneglycol (CAS 6239456-72-5) were prepared by reflux as previously reported.[14, 15] Paper supported porphyrin indicators were prepared using a dip and dry technique. For a 5 x 33 $\mathrm{cm}$ swatch, $0.4 \mathrm{mM}$ porphyrin in water (total volume $6 \mathrm{~mL}$ ) was used. The paper support (WypAll X60) was pulled through this solution and allowed to dry slightly before being pulled through the solution again. This was repeated until all porphyrin solution had been deposited (typically three cycles). Samples were then dried at $100^{\circ} \mathrm{C}$ before storing in the dark in sealed plastic bags. This is a modification of the procedure described previously for preparation of the indicator materials.[14]

The prototype reflectance instrument utilized low cost, commercially available color sensing breakout boards from Parallax, Inc. (model TCS3200-DB, Rocklin, CA), providing a color lightto-frequency integrated circuit from AMS (model TCS3200, Plano, TX), a pair of white LEDs, and an adjustable lens. The device was previously described in detail.[14] Briefly, six of the breakout boards were used with a customized multiplex platform in which the boards were mounted using in-house developed holders made from chemically resistant Delrin plastic 
(McMaster-Carr, Princeton, NJ). The indicator support provides a lip on the bottom that can sit on top of a Petri dish or bottle (Figure 1). The device output consists of a stream of digital pulses proportional to the intensity of the color being measured. A custom printed circuit board (PCB) interfaces with and controls the six sensors. The PCB uses an Atmel ATMega microcontroller (Atmel Corporation, San Jose, CA) to regulate the timing of events, count pulses, and report the results to a computer. Communications between the instrument and the computer are via USB; power is supplied through a dc barrel jack. A LabWindows developed software-based graphical user interface (GUI) communicates with the PCB firmware through simple ASCII commands.

Target exposure was completed using $200 \mathrm{~mL}$ Nalgene bottles containing a solution volume of $30 \mathrm{~mL}$. Target solutions consisted of deionized water; dilutions of $30 \%$ hydrogen peroxide; dilutions of sulfuric, hydrochloric, and nitric acid; and solvents such as ethanol and acetone. $\mathrm{H}_{2} \mathrm{O}_{2}$ solutions of $3,1.2,0.3,0.15$, and $0.06 \%$ in water provided maximum initial headspace concentrations of $8.45,3.38,0.84,0.42$, and $0.17 \mathrm{ppm}$, respectively. Solutions were prepared and capped for 2 to $4 \mathrm{~h}$ to allow for headspace equilibration prior to exposure of indicators. Data was collected for a minimum of five minutes prior to target exposure to establish a baseline for the indicators. For this measurement, bottles containing $30 \mathrm{~mL}$ of water were used as the control solutions. Exposure was initiated by exchanging water containing bottles for those containing target. An alternative approach accomplished target exposure by placing the indicator supports over empty Petri dishes for pre- and post-exposure measurements. The exposure measurements were completed by placing the holder over a Petri dish $(60 \mathrm{~mm}$; total volume $57 \mathrm{~mL})$ containing $1 \mathrm{~mL}$ of warmed target solution $\left(10 \mathrm{~min}\right.$ in oven at $\left.60^{\circ} \mathrm{C}\right)$.

\section{Results and Discussion.}

The titanyl indicators were evaluated using the prototype reflectance sensor with target (30 $\mathrm{mL}$ ) in $200 \mathrm{~mL}$ Nalgene bottles. This experiment is significantly different from those described in the original report.[13] Initial characterization utilized a sealed $9 \mathrm{~L}$ headspace over $1 \mathrm{~L}$ of target solution with a fan generating impacting air flow. Under these conditions, the target content at even the lowest utilized concentrations ( $0.1 \mathrm{ppm}$ vapor) would not change over the course of the measurement. The experiments of the current study were not conducted in a sealed headspace nor did they utilize a large target excess; concentrations of target were expected to change over time. Ambient temperatures were between 24 and $27^{\circ} \mathrm{C}$ with relative humidity between 43 and 55\%. Though the prototype device reports red, green, and blue (RGB) color values (Figure 2, Panel A), changes observed for the titanyl indicators were much more dramatic for the blue channel than for the red or green. The data has been normalized using the average value from the pre-exposure measurement to account for sensor to sensor variation in the data. In Figure 2 (Panel B), we report the changes in the blue channel over time following exposure of indicators to various concentrations of hydrogen peroxide. The rates of change in reflectance for the titanyl indicators (blue and green values only) were found to be concentration dependent with saturation of the indicator occurring at $\sim 9.5 \mathrm{~h}$ for the $3 \%$ target solution and $\sim 15.5 \mathrm{~h}$ for the $1.2 \%$ target solution. Measurements were continued to $66.5 \mathrm{~h}$; none of the lower target concentrations resulted in indicator saturation. (Additional results provided in the Supplementary Material)

Previous work with the prototype sensor utilized porphyrin indicators and focused on chemosorptive interactions, $[14,16]$ often of a reversible nature. The titanyl compounds utilize a reactive interaction (non-reversible) with the peroxide vapor. Representative porphyrin indicators were evaluated for comparison to the titanyl materials (Figure 3). The CoDIX indicator showed significant changes in reflectance upon exposure to hydrogen peroxide while 
changes in CuDIX reflectance were smaller and NiDIX did not respond. The changes in CoDIX and CuDIX were found to be reversible when target was removed from the indicator environment. Porphyrin indicators were also found to respond to the presence of sulfuric, nitric, and hydrochloric acid (additional results provided in the Supplementary Material). As previously reported, these DIX variants are also sensitive to alcohol vapors.[14] The reflectance of the titanyl indicators does not change upon exposure to acids or alcohols; they provide unique indication of the presence of peroxide vapor. This specificity may offer a significant advantage depending on the application, especially if peroxide vapor is a primary or high value target.

There are other aspects that should be considered when designing a sensor system. The differences between the porphyrin and titanyl indicators serve to illustrate some of these points. The titanyl indicators are dosimetry type reporters; that is, the reported signal is an integration of exposure concentrations and durations. These indicators will reach a saturation point after which they are no longer useful for detection of target. The porphyrin indicators, on the other hand, respond to increasing and decreasing concentrations with reversible changes in reflectance. This provides the potential for continuous or long term applications but makes them unsuitable for reporting on total exposure. The titanyl indicators could be regenerated through hydrolysis in basic water followed by reaction with oxalic acid; however, treatment of the paper supported indicator with liquids will result in migration. Regeneration would likely also be more expensive than replacement of the indicator.

A further consideration in characterization of indicator materials for sensor applications is quantification of target concentrations. The reactive nature of the titanyl indicators was originally described as providing the potential for determination of concentrations of peroxide vapor on the basis of rates of change in indicator color.[13] This description should be qualified to be specific for situations under which the sampled space is stagnant and of sufficient volume to prevent reactant depletion. The rate equations developed under the original work cannot be applied to the experimental conditions utilized in the current study. Time is also an important consideration for the proposed type of analysis. These are not necessarily a realizable set of conditions. Dramatic fluctuations in target concentration would be expected for point sensors in an environmental sensing scenario. $[17,18]$

\section{Acknowledgements.}

This research was sponsored by the U.S. Naval Research Laboratory (WU\# 69-6594). R. Neblett was supported through an Office of Naval Research (ONR) sponsored summer research internship. Participation of R. Liu was through the US Navy Science and Engineering Apprenticeship Program (SEAP). The views expressed here are those of the authors and do not represent those of the U.S. Navy, the U.S. Department of Defense, or the U.S. Government.

Scheme 1. Modification of titanyl indicator by peroxide resulting in yellow color.

Figure 1. Photograph of the prototype sensor system with fresh (A) and exposed (B) titanyl indicators and $(\mathrm{C})$ the CoDIX indicator.

Figure 2. Response of paper supported titanyl indicators to hydrogen peroxide. (A) Shown here are the as reported red, green, and blue color values (dark to light) for an indicator swatch before and during exposure to the vapor evolving from a solution of $3 \% \mathrm{H}_{2} \mathrm{O}_{2}$ in water (dashed line marks beginning of exposure period). (B) The normalized blue color values are reported for 
indicator swatches before and during exposure to vapor evolving from water (black) and 3, 1.2, $0.3,0.15$, and $0.06 \%$ (dark to light) solutions of $\mathrm{H}_{2} \mathrm{O}_{2}$ in water.

Figure 3. Response of paper porphyrin indicator to hydrogen peroxide. (A) Shown here are the normalized red, green, and blue color values (dark to light) for a CoDIX indicator swatch before and during exposure to the vapor evolving from a solution of $3 \% \mathrm{H}_{2} \mathrm{O}_{2}$ in water (dashed line marks beginning of exposure period). (B) The normalized blue color values are reported for CoDIX indicator swatches before and during exposure to vapor evolving from water (black), 3 , $1.2,0.3$, and $0.15 \%$ (dark to light) solutions of $\mathrm{H}_{2} \mathrm{O}_{2}$ in water.

\section{References.}

[1] R.M. Burks, D.S. Hage, Current trends in the detection of peroxide-based explosives, Analytical and Bioanalytical Chemistry, 395(2009) 301-13.

[2] M.E. Germain, M.J. Knapp, Optical explosives detection: from color changes to fluorescence turn-on, Chemical Society Reviews, 38(2009) 2543-55.

[3] T. Krawczyk, S. Baj, Review: Advances in the determination of peroxides by optical and spectroscopic methods, Analytical Letters, 47(2014) 2129-47.

[4] R. Bailey, D.F. Boltz, Differentical spectrophotometric determination of hydrogen peroxide using 1,10-phenanthroline and bathophenanthroline, Analytical Chemistry, 31(1959) 117-9.

[5] C. Matsubara, K. Kudo, T. Kawashita, K. Takamura, Spectrophotometric determination of hydrogen-peroxide with titanium 2((5-bromopyridyl)azo)-5-(N-propyl-N-

sulfopropylamino)phenol reagent and its application to the determination of serum glucose using glucose-oxidase, Analytical Chemistry, 57(1985) 1107-9.

[6] K. Takamura, C. Matsubara, Versatility of the Titanium(IV) - Porphyrin reagent for determining hydrogen peroxide, Bulletin of the Chemical Society of Japan, 76(2003) 1873-88.

[7] G.M. Eisenberg, Colorimetric determination of hydrogen peroxide, Industrial and Engineering Chemistry-Analytical Edition, 15(1943) 327-8.

[8] B.D. Patterson, E.A. Macrae, I.B. Ferguson, Estimation of hydrogen-peroxide in plantextracts using titanium (IV), Analytical Biochemistry, 139(1984) 487-92.

[9] C. Matsubara, N. Kawamoto, K. Takamura, Oxo 5,10,15,20-tetra(4-pyridyl)porphyrinato titanium(IV) - An ultra-high sensitivity spectrophotometric reagent for hydrogen-peroxide, Analyst, 117(1992) 1781-4.

[10] F.I. Bohrer, C.N. Colesniuc, J. Park, I.K. Schuller, A.C. Kummel, W.C. Trogler, Selective detection of vapor phase hydrogen peroxide with phthalocyanine chemiresistors, Journal of the American Chemical Society, 130(2008) 3712-3.

[11] R.M. Sellers, Spectrophotometric determination of hydrogen peroxide using potassium Titanium (IV) oxalate, Analyst, 105(1980) 950-4.

[12] J. Muhlebach, K. Muller, G. Schwarzenbach, The peroxo complexes of titanium, Inorganic Chemistry, 9(1970) 2381-90.

[13] M. Xu, B.R. Bunes, L. Zang, Paper-Based Vapor Detection of Hydrogen Peroxide: Colorimetric Sensing with Tunable Interface, Acs Applied Materials \& Interfaces, 3(2011) 6427.

[14] B.J. Johnson, J.S. Erickson, J. Kim, A.P. Malanoski, I.A. Leska, S.M. Monk, et al., Miniaturized reflectance devices for chemical sensing, Measurement Science \& Technology, 25(2014) 095101 (10pp). 
[15] B.J. Johnson, N.E. Anderson, P.T. Charles, A.P. Malanoski, B.J. Melde, M. Nasir, et al., Porphyrin-embedded silicate materials for detection of hydrocarbon solvents, Sensors, 11(2011) 886-904.

[16] L. Feng, C.J. Musto, J.W. Kemling, S.H. Lim, K.S. Suslick, A colorimetric sensor array for identification of toxic gases below permissible exposure limits, Chem Comm, 46(2010) 2037-39. [17] P.R. Best, K.E. Lunney, C.A. Killip, Statistical elements of predicting the impact of a variety of odour sources, Water Sci Technol, 44(2001) 157-64.

[18] K. Obenschain, J. Boris, G. Patnaik, Using CT-Analyst to Optimize Sensor Placement, Defense and Security, (2004) 14-20. 


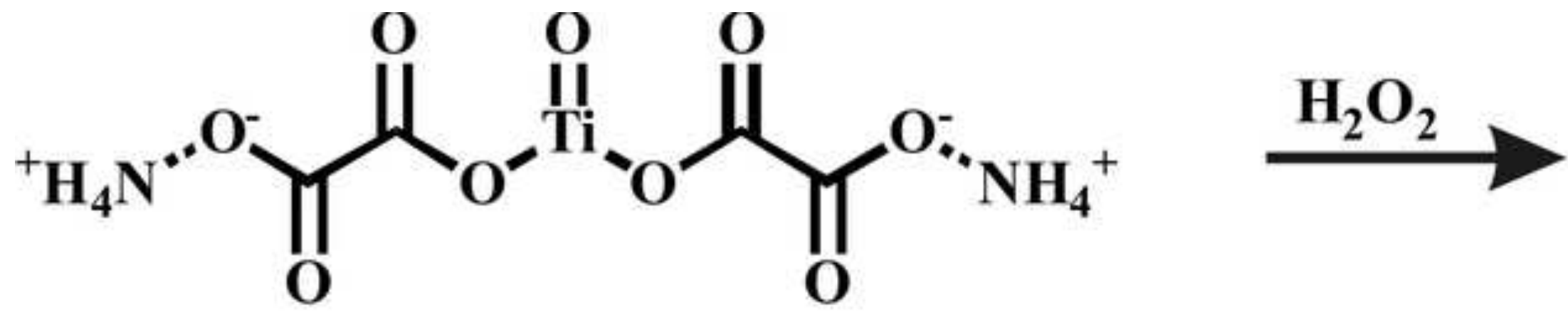

colorless

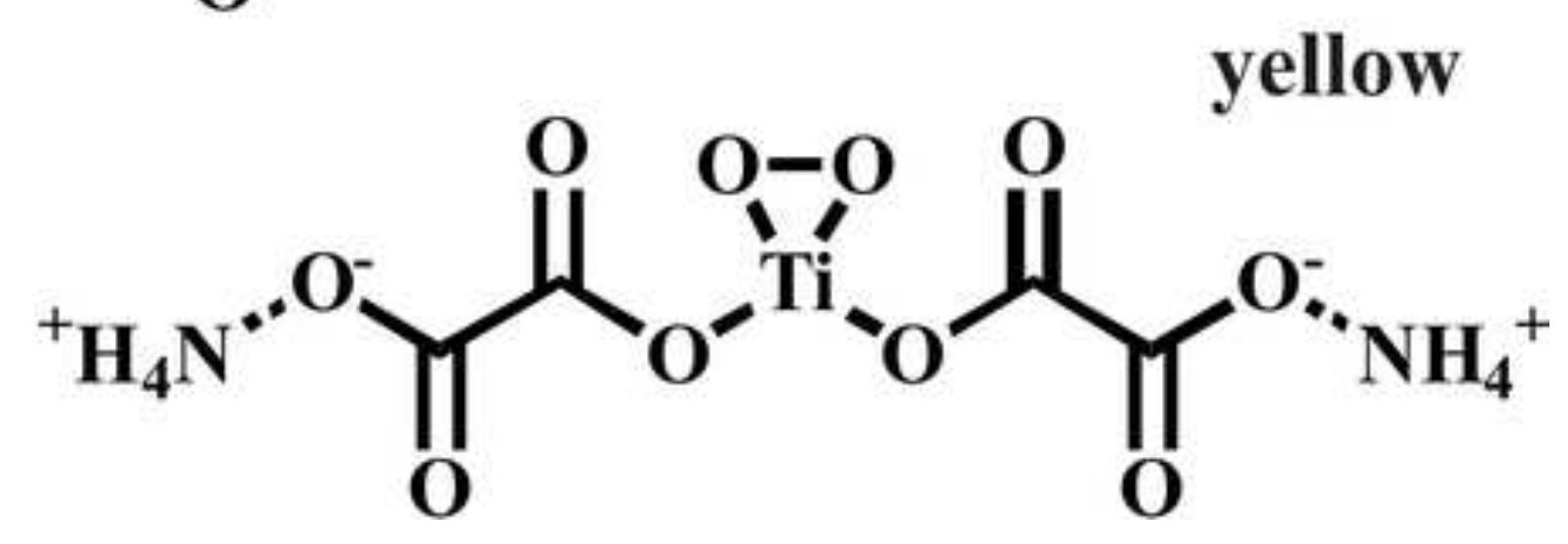



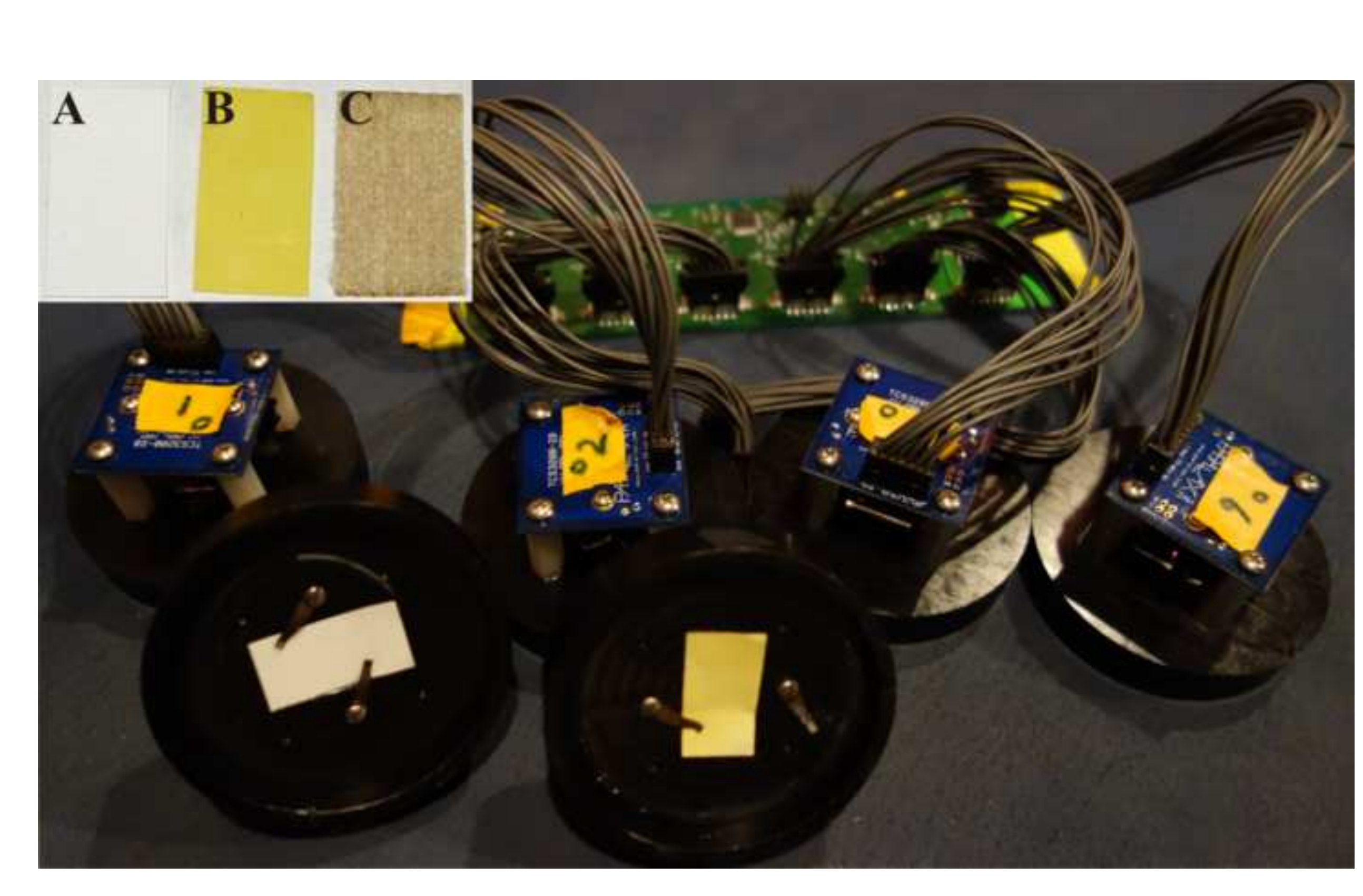

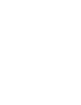

(

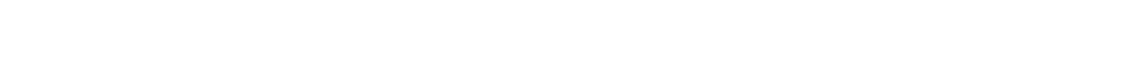




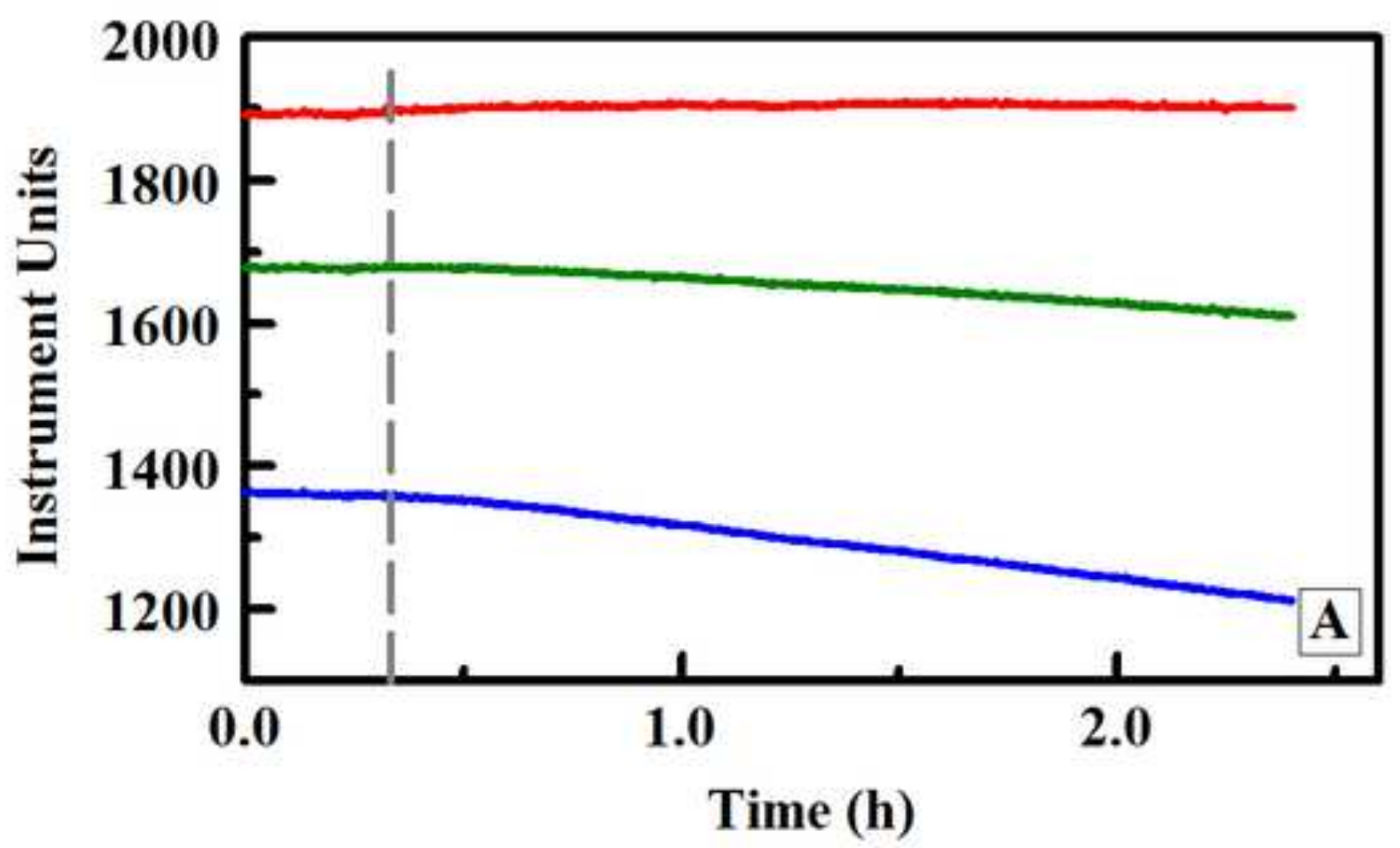

Time (h)

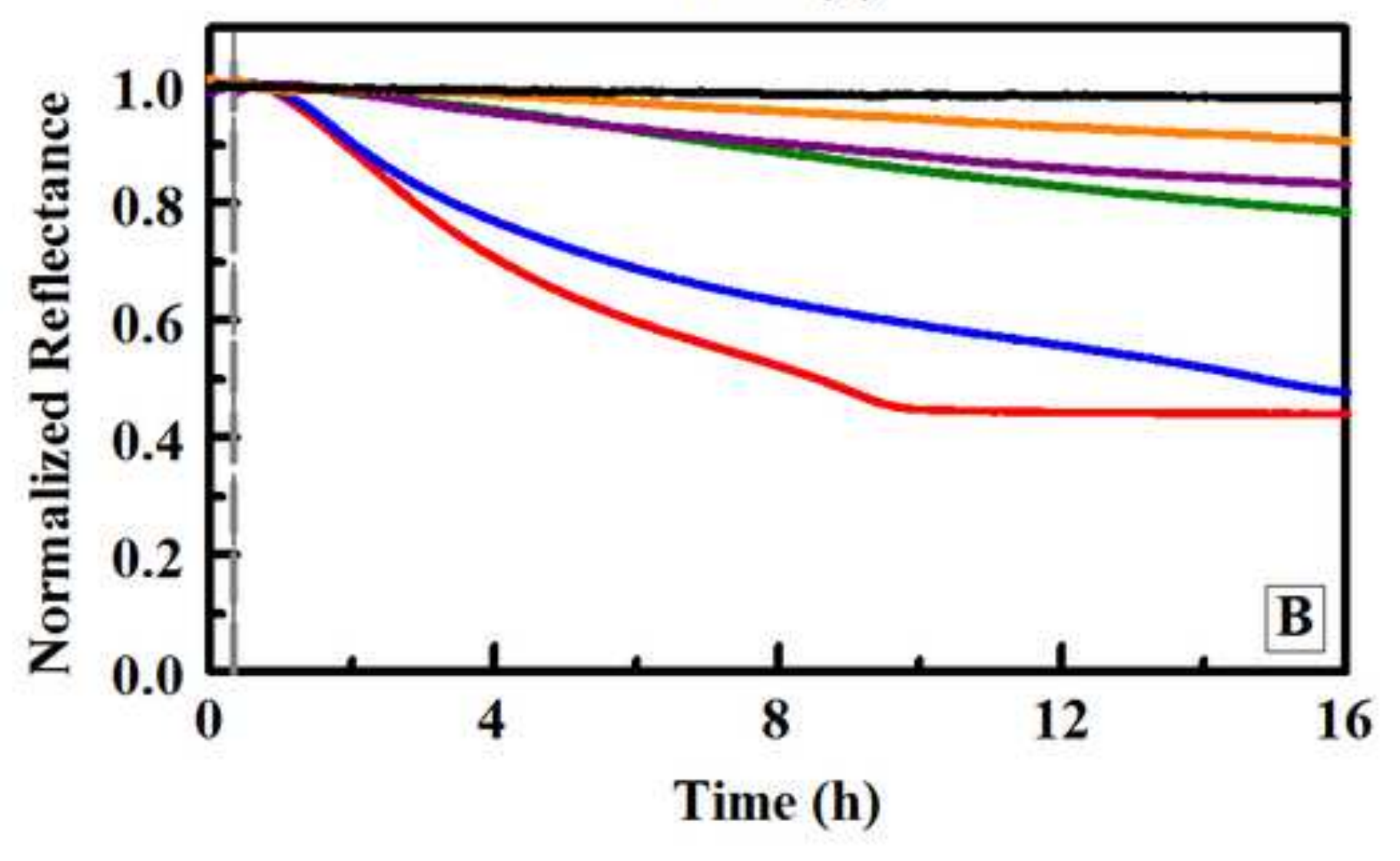

Figure 2 color
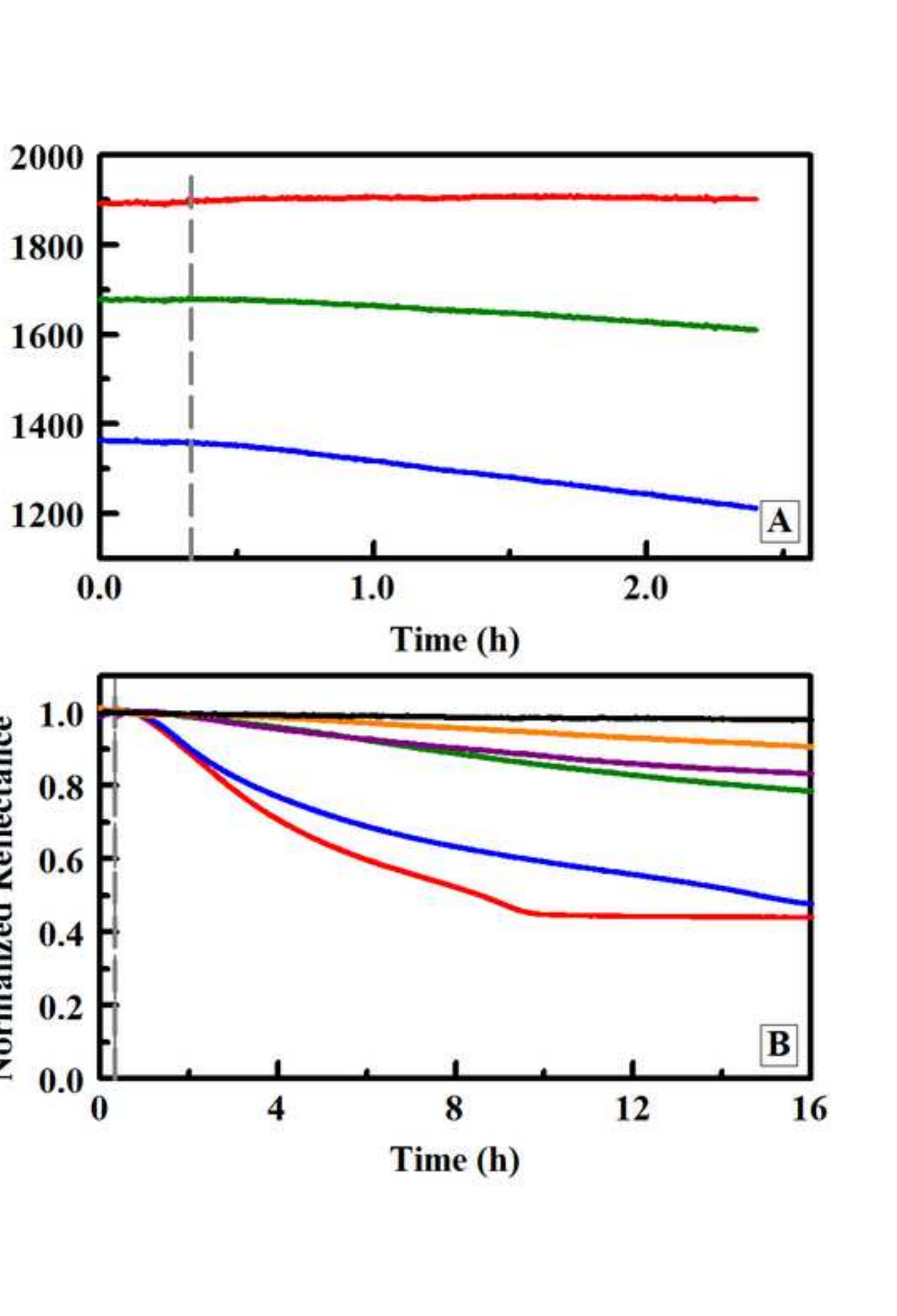

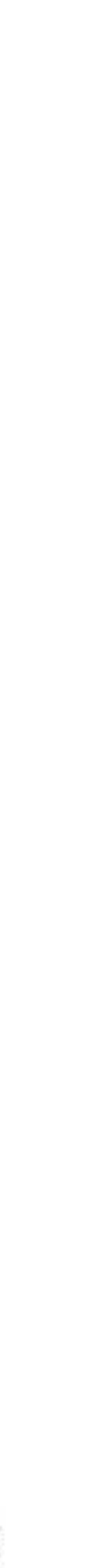

(

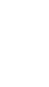



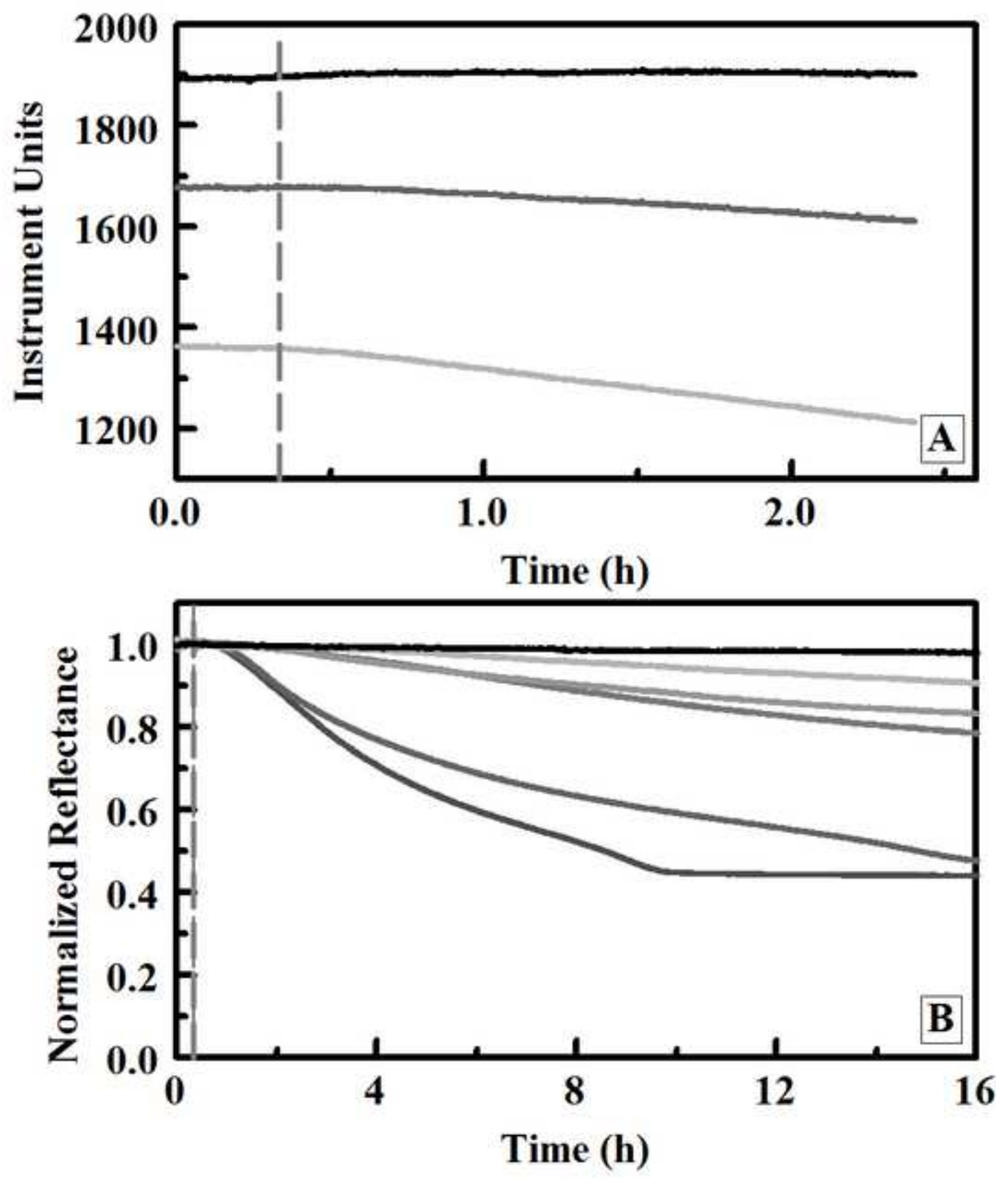

Figure 2 gray scale

Time (h) 
Figure 3 color

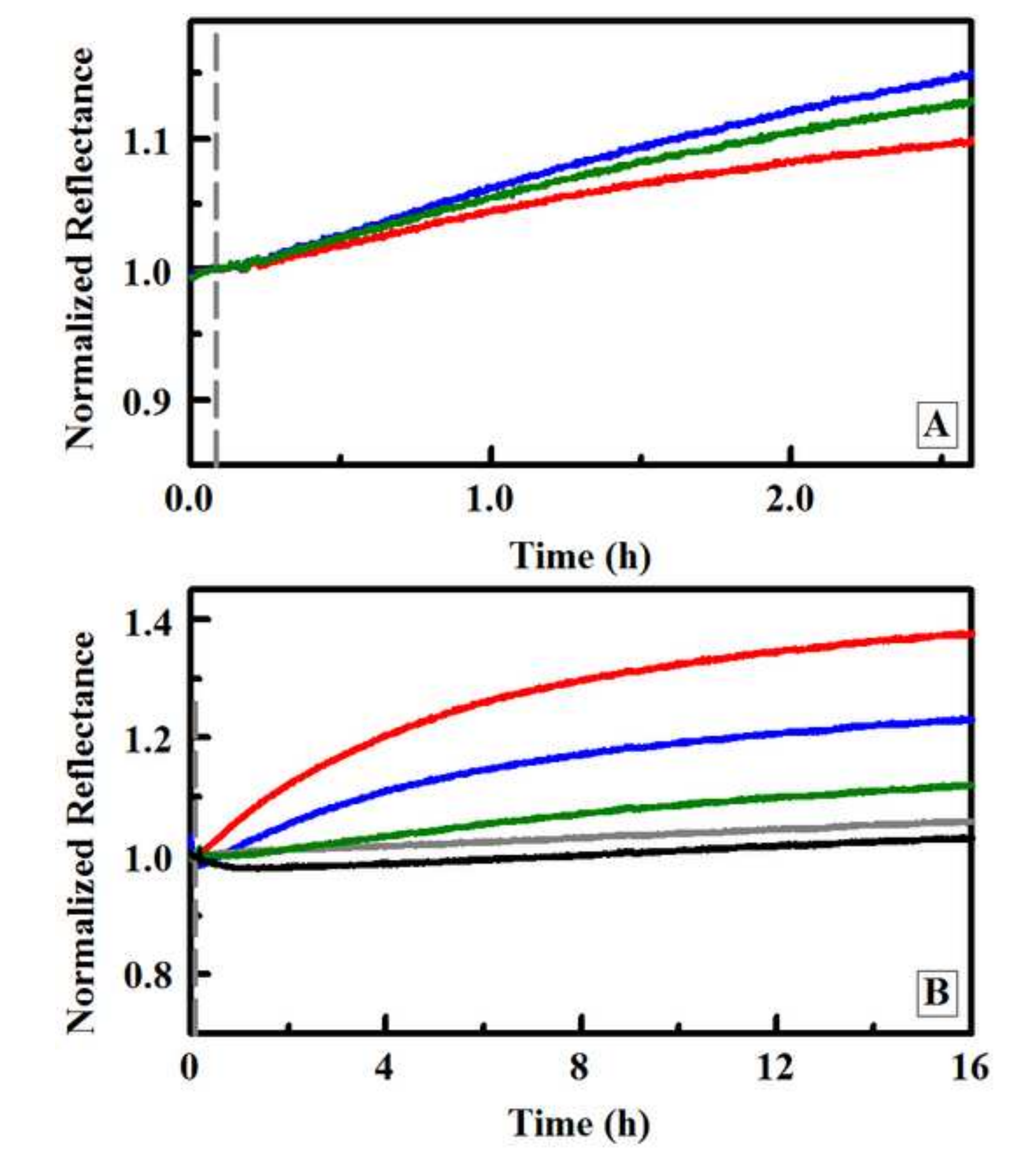

Time (h)

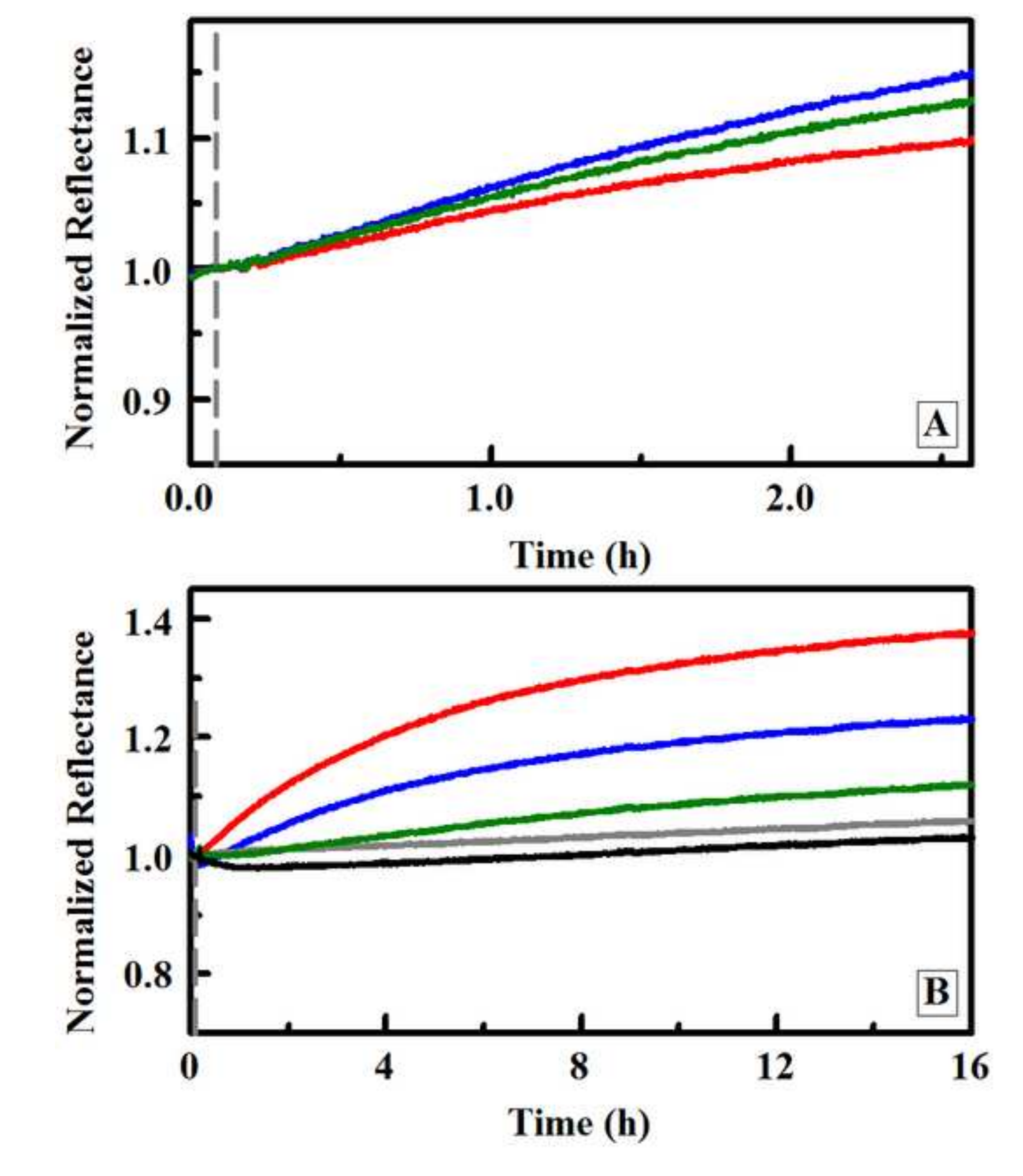


Brandy Johnson received a Doctoral degree in Photonics (2004) from Oklahoma State University based on research directed at the application of porphyrins in chemical detection. Since joining the Naval Research Laboratory's Center for Bio/Molecular Science and Engineering in 2004, she has pursued research into distributed sensing, environmental monitoring, and selfdecontaminating materials.

Ray Liu is currently a student enrolled at Thomas Jefferson High School for Science and Technology, where he has pursued advanced coursework in chemistry, physics, mathematics, and computer science. He has interned at the Naval Research Laboratory for two consecutive summers and aspires to be an engineer.

Robert Neblett is a rising senior in the Biology Department at Howard University. He spent the summer of 2015 as an intern at the Naval Research Laboratory. He plans to pursue a graduate degree in biology with his current interests lying at the intersection of science and business.

Dr. Anthony P. Malanoski received a Doctoral degree (1999) in Chemical Engineering at the University of Massachusetts Amherst, MA. After postdoctoral work at the University of New Mexico and Sandia National Labs in New Mexico, he joined the Naval Research Laboratory (2003). His current research interests include thermodynamic and kinetic reaction modelling of nano-scale systems and metagenomic bioinformatics.

David Stenger earned his Ph.D. in Biophysics at SUNY at Buffalo in 1989. He is a Senior Scientist in the Center for Bio/Molecular Science and Engineering, Naval Research Laboratory, Washington, DC. His research focuses on distributed sensors, autonomous systems, and advanced diagnostics.

Jeffrey Erickson earned his PhD degree in Chemical and Biomolecular Engineering at the Johns Hopkins University in 2004. He is currently working as an engineer at the Naval Research Laboratory. His research focuses on incorporating bench-scale research developments into portable devices and bringing them from the laboratory into the field.

Miao Xu is currently a postdoctoral fellow in Dr. Ling Zang's group, Department of Materials Science and Engineering, University of Utah. He received his B.S. in chemistry and M.S. in inorganic chemistry from Fudan University and his Ph.D. in Materials Science from University of Utah. His research interests are optoelectronic nanodevices and fluorescent sensors.

Ling Zang is a USTAR professor at University of Utah, affiliated with Department of Materials Science and Engineering, Department of Chemistry, and Nano Institute of Utah. He received his B.S. in chemistry from Tsinghua University and Ph.D. in chemistry from the Chinese Academy of Sciences. His current research focuses on nanoscale imaging and molecular probing, organic semiconductors and nanostructures, optoelectronic sensors and nanodevices.

Martin Moore received his MS degree in chemistry from Youngstown State University in 1998. He is currently a research chemist at the Naval Research Laboratory focusing on synthesis of small molecules and their analytical characterization. 